\title{
Thermodynamics in the Evolution of the Dark Universe
}

\author{
Carlos A. Melendres \\ The SHD Institute, Davis, CA, USA \\ Email: camelendres@SHDInstitute.org
}

How to cite this paper: Melendres, C.A. (2021) Thermodynamics in the Evolution of the Dark Universe. Journal of Modern Physics, 12, 1527-1544.

https://doi.org/10.4236/jmp.2021.1211092

Received: June 21, 2021

Accepted: September 10, 2021

Published: September 13, 2021

Copyright $\odot 2021$ by author(s) and Scientific Research Publishing Inc. This work is licensed under the Creative Commons Attribution International License (CC BY 4.0).

http://creativecommons.org/licenses/by/4.0/

\begin{abstract}
We present a model of the universe based on the theory that space consists of energy quanta. We use the thermodynamics of an ideal gas to elucidate the composition, accelerated expansion, and the nature of dark energy and dark matter without an Inflation stage. From wave-particle duality, the space quanta can be treated as an ideal gas. The universe started from an atomic size volume at very high temperature and pressure. Upon expansion and cooling, phase transitions occurred to form fundamental particles, and matter. These nucleate and grew into stars, galaxies, and clusters due to gravity. From cooling data, a thermodynamic phase diagram of cosmic composition was constructed which yielded a correlation between dark energy and the energy of space. Using Friedmann's equations, our model fits well the Williamson Microwave Anisotropy Platform (WMAP) data on cosmic composition with an equation of state parameter, $w=-0.7$. The dominance of dark energy started at $7.25 \times 10^{9}$ years, in good agreement with Baryon Oscillation Spectroscopic Survey (BOSS) measurements. The expansion of space can be attributed to a scalar space field. Dark Matter is identified as a plasma form of matter similar to that which existed before recombination and during the reionization epoch. The expansion of the universe was adiabatic and decelerating during the first 7 billion years after the Big Bang; it accelerated thereafter. A negative pressure for Dark Energy is required to sustain it; this is consistent with the theory of General Relativity and energy conservation. We propose a mechanism for the acceleration as due to the consolidation of matter to form Black Holes and other massive compact objects. The resulting reduction in gravitational potential energy feeds back energy for the acceleration. It is not due to a repulsive form of gravity. Our Quantum Space model fits well the observed behavior of the universe and resolves the outstanding questions in Inflationary Big Bang Theory.
\end{abstract}

\section{Keywords}

Thermodynamics, Quantum Space Model, Dark Universe, Composition 
and Expansion, Phase Diagram, Spaceons, Dark Energy, Dark Matter,

Cosmological Constant, Inflation

\section{Introduction}

The evolution of the universe is a subject of great interest in Physics, Astronomy, Cosmology, Astrochemistry, and Science in general. It is intimately related to the expansion and composition of the universe. Our universe is essentially dark, consisting of $71 \%$ dark energy, $24 \%$ dark matter; it has only $5 \%$ ordinary matter, of which $0.5 \%$ is luminous. The nature of dark matter and dark energy remains unknown. Dark energy is theorized to cause the expansion of the universe, dark matter is thought to hold the galaxies together. They remain rather "mysterious", along with Inflation. Enormous scientific efforts are expended in order to understand them.

The evolution of the universe is intimately related to its expansion and that of space. The nature of space is unknown but much debated [1]. It is generally viewed like a canvas where nature's landscape and events are portrayed; it is then treated geometrically and mathematically as a surface in 4-dimensional spacetime in Einstein's Theory of General Relativity. Here, we present a more descriptive physical model of space based on the theory that it is a quantized dynamical entity. We theorize that it is the cosmic fluid that actively participates in the evolution of the universe, along with matter and radiation. The mechanism of evolution proceeds via well-known processes. Our Quantum Space model provides a rational explanation for the accelerated expansion of the universe due to dark energy and the nature of dark matter, without the need for a Theory of Inflation.

\section{A Model of Space and Cosmic Evolution}

It has been thought that space is not really empty, that "vacuum" contains virtual particles which pop in and out of existence, and is used to explain the Casimir effect [2]. We take the view that space is associated with energy and is quantized. Space consists of energy quanta that propagate as waves described by the Planck quantum energy expression:

$$
E=h c / \lambda
$$

The symbols have their usual meaning: $E$, is the energy; $\lambda$, the wavelength, and $h$, the Planck constant; we assume the velocity of spaceons to be the same as that of light, $c$. In terms of equivalent volume, we could think of spaceons as spherical waves, or consider them like bubbles, such that

$$
E=(\pi / 3 V)^{1 / 3} h c
$$

where $V$ is the volume of a sphere, equal to (4/3) $\pi r^{3}$ and $r=\lambda / 2$. The expressions above define the equivalence of space (in terms of the dimensions $\lambda$ or volume 
$V)$, and energy, in analogy to the relation between energy and mass, given by Einstein's equation, $E=m c^{2}$. Thus, energy is inherent in space and space in energy. We will call the units of space, "spaceons". They can be thought of as the carrier of energy and weave the fabric of the universe. From wave-particle duality, the spaceons can be thought of as an ideal gas which obeys the equation of state [3],

$$
P V=N_{0} K_{\beta} T
$$

$P$ is the pressure of the gas, $V$ the volume, $T$ the temperature, $N$ the Avogadro number and $K_{\beta}$ the Boltzman constant. With this theory we can model the gross features of the evolution of the universe as follows:

The universe started (at time, $t=0$ ) as a very small volume of an ideal gas (the spaceons), at an extremely high pressure and temperature. For example, an Avogadro number of gas particles occupying a volume of $4.2 \times 10^{-36} \mathrm{~m}^{3}$ (a spherical wave of $1 \mathrm{~A}^{0}$ diameter), at a pressure of $1.976 \times 10^{66} \mathrm{~Pa}$ and a temperature of $10^{32} \mathrm{~K}$. In this initial state of its birth, the universe consisted of "hot" spaceons and presumably radiation. We shall refer to this period as the Quantum Space Epoch. The spaceons then expanded and cooled as they propagate. In the process of cooling to appropriate threshold temperatures, phase transitions occurred resulting in the formation of fundamental particles, nuclei, and atoms. From the matter formed, gravitation caused the formation of galaxies and stars; these clumped to form clusters, local groups and superclusters. The mechanisms of matter formation follow those of the Standard Model of Particle Physics; the nucleation and growth of atoms to stars and superclusters are not completely understood as yet. The universe continued to expand until the present time. The various epochs of the evolution of the universe, in our model, are similar to those of the Inflationary Big Bang Theory (or Lambda Cold Dark Matter model) [4] [5] [6], but without the need for an Inflation stage.

\section{Results and Discussion}

\subsection{Composition of the Universe}

Several methods are used to determine the composition of the universe. Results of the Wilkinson Microwave Anisotropy Probe (WMAP) satellite studies [7] gave the composition shown in Table 1 soon after the Big Bang and at present.

The composition during the cosmic evolution has also been documented [4] [5] [6]. Table 2 shows this as a function of temperature $(T)$ at various times and Cosmic Epochs, together with the cooling information (Composition vs $T, P, t$ ).

At temperatures above $10^{12} \mathrm{~K}$ and time $<10^{-12} \mathrm{sec}$, the universe was a primeval hot gas of spaceons and presumably radiation, in equilibrium. Upon expansion and cooling, fundamental particles (quarks, leptons, hadrons, protons, neutrons, electrons) were created. On further cooling to about $10^{9} \mathrm{~K}$, nucleosynthesis occurred to produce nuclei of $\mathrm{H}, \mathrm{He} \mathrm{Li}$ and $\mathrm{D}$. At about $10^{8} \mathrm{~K}$, a plasma phase was formed which consisted of electrons and positive ions of $\mathrm{H}$ and He. Radiation 
Table 1. Composition of the universe.

\begin{tabular}{ccc}
\hline At the Big Bang & & At Present $(13.8$ billion years after $)$ \\
\hline Dark Matter & $63 \%$ & $24 \%$ \\
Dark Energy & - & $71.4 \%$ \\
Ordinary Matter & $12 \%$ & $4.6 \%$ \\
Neutrinos & $10 \%$ & - \\
Photons & $15 \%$ & - \\
\hline
\end{tabular}

Table 2. Timeline of the universe and cooling data during cosmic evolution.

\begin{tabular}{|c|c|c|c|}
\hline $\begin{array}{c}\text { Time }(\mathrm{t}) \text { after Big } \\
\text { Bang)/(Cosmic Epoch) }\end{array}$ & $\begin{array}{c}\text { Temperature } \\
(\mathrm{K})\end{array}$ & Pressure $(\mathrm{Pa})$ & Composition \\
\hline $\begin{array}{l}<10^{-12} \mathrm{sec} \\
\text { (Quantum Space Epoch) }\end{array}$ & $>10^{12}$ & $>10^{32}$ & 1) Hot spaceons, radiation (?) \\
\hline $\begin{array}{l}10^{-12} \text { to } 0.02 \mathrm{sec} \\
\text { (Quark, Hadron, Lepton, } \\
\text { Electrons, Protons, } \\
\text { Neutrons) }\end{array}$ & $10^{11}-10^{9}$ & $10^{32}-10^{27}$ & $\begin{array}{l}\text { 2) Radiation, quarks, gluons, } \\
\text { "gluon soup", fundamental } \\
\text { particles }\end{array}$ \\
\hline $\begin{array}{l}0.02 \text { to } 300 \mathrm{sec} / \\
\text { (Nucleosynthesis) }\end{array}$ & $10^{9}-10^{8}$ & $10^{27}-10^{15}$ & $\begin{array}{l}\text { 3) Nuclei of } \mathrm{H}, \mathrm{He}, \mathrm{D}, \mathrm{Li} \\
\text { (plasma) + radiation }\end{array}$ \\
\hline $\begin{array}{l}3.8 \times 10^{5} \text { to } 10^{9} \mathrm{yrs} /(\text { Dark } \\
\text { Ages to Matter } \\
\text { dominated) }\end{array}$ & $3 \times 10^{3}-4$ & $10^{-11}-10^{-22}$ & $\begin{array}{l}\text { 4) Matter in galaxies, stars, } \\
\text { planets, gases, plasma, solid }\end{array}$ \\
\hline $\begin{array}{l}10^{9} \text { to } 13.8 \times 10^{9} \mathrm{yrs} / \\
\text { (Dark Energy dominated) }\end{array}$ & $4-2$. & $10^{-22}$ & 5) Dark Energy \\
\hline
\end{tabular}

(photons, neutrinos) was ever present and dominated the early epoch of the universe. Further cooling of the plasma until about $3 \times 10^{3} \mathrm{~K}$ resulted in recombination of electrons with positive ions of $\mathrm{H}$ and $\mathrm{He}$, converting the plasma to gaseous elements. As the universe continued to expand and cool, matter continued to form and grow through the action of gravity to become stars, galaxies, and clusters. The universe cooled to $2.7 \mathrm{~K}$ as indicated by the Cosmic Microwave background (CMB). The primary constituents of the universe are: gases $(\mathrm{H}, \mathrm{He})$, plasma of electrons, protons, and He ions, ordinary matter (gasses, solids, dust, in stars, galaxies, clusters, intergalactic space), radiation, and spaceons (gas at all temperatures).

Thermodynamics is a very powerful method for obtaining compositional information [3] [8]. The timeline of the universe is shown in Table 2. We also show its cooling rate, i.e., composition as a function of temperature, pressure, and time, which we have derived. From the latter, together with WMAP data on the constituents of the universe (Table 1), we constructed a thermodynamic phase diagram [3] [8] for our model universe. Such diagrams are routinely used in Chemistry, Metallurgy and Geophysics; though normally confined to the components of matter. The equivalence of matter and energy, allows us to do the same for the universe as a whole system with matter, energy and radiation as 
components.

The result for our model universe is shown in Figure 1. Note that the figure is schematic and the temperature is not drawn to scale in order to highlight the phases formed. We only emphasized the points at which the phases are formed because the exact pressure dependence is not fully known. The major phases are: radiation, matter (IV, solid, gasses, plasma formed in galaxies, stars, clusters), a plasma phase (III) formed immediately after nucleosynthesis, dark energy (V) and spaceons (gas). The broken lines indicate the overlap between various phases during the process of formation. While dark energy (spaceons) were present at the beginning of the universe, it's phase transition is indicated only at the time when it became the major component of the universe. Dark matter is underlined since its nature is still unknown and unassigned. All phases are in contact with spaceons at all epochs of cosmic evolution, as indicated. This is as it should be since space is in contact with all elements of the universe at all times. This results in an isotropic and homogeneous universe so that there is no need for an inflationary stage. At extremely high temperatures and pressures (right end), fundamental particles and radiation are indistinguishable from the hot spaceons. At low temperature/pressure (left end), dark energy overlaps with the cold spaceons which may further transition to another phase. These end points are critical points, where 2 phases co-exist. (For water, at the critical temperature of $647 \mathrm{~K}$ and pressure of $2.2064 \times 10^{7} \mathrm{~Pa}$, liquid water is indistinguishable from its vapor phase).

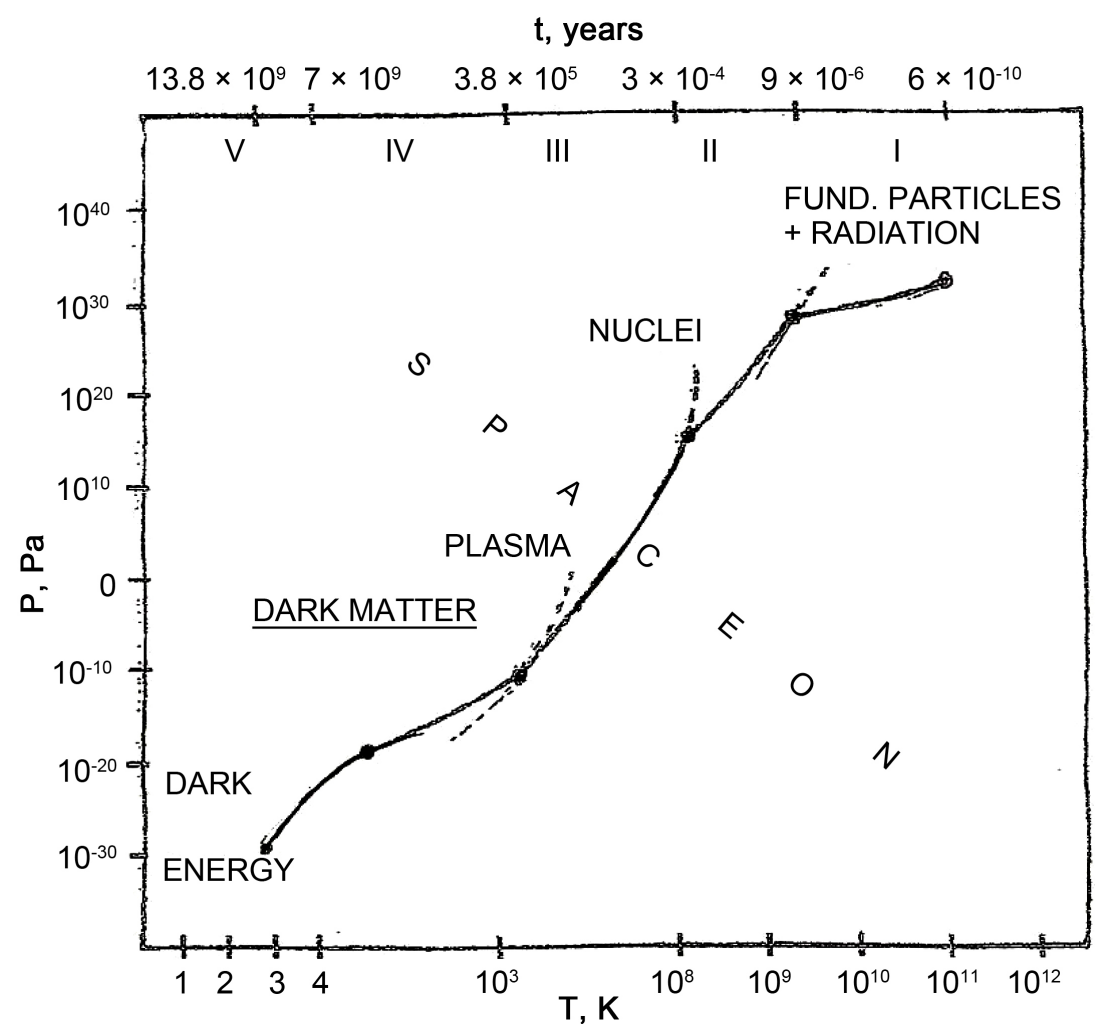

Figure 1. Schematic phase diagram of the model universe with spaceons. 


\subsection{Dark Energy and the Expansion of the Universe}

Dark energy constitutes $71 \%$ of our universe. It is hypothesized to be an unknown form of energy that permeates all of space uniformly. It is invisible and difficult to study because it does not interact with radiation, hence cannot be investigated spectroscopically. It only interacts with gravity. Its density is very low, less than ordinary and dark matter; it converts to dark matter, was less in the past than at present, and it is thought to function like an anti-gravity force which causes the accelerated expansion of the universe [9] [10] [11].

Based on our model, the expansion of the universe could be thought of as the expansion of spaceons into the Void ("nothingness"). It can be thought of as driven by the pressure and temperature differential of the hot high energy state (high $\mathrm{T}$, short $\lambda \mathrm{s}$ ) and the cold state in the $\operatorname{Void}(T \approx 0 \mathrm{~K}, \lambda \approx \infty)$. It is an inherent property of space that it needs to expand in order to attain a lower energy state. The expansion may be viewed from the standpoint of Quantum Field Theory [12], as arising from a force that is associated with a field. We call the latter the space field from which emanate space quanta, the spaceons. It is a scalar field, often referred to in the literature as "Quintessence". It has been theorized to be the substance which comprises Dark Energy [10].

One can see from our thermodynamic phase diagram (Figure 1) that dark energy is a phase that overlaps with the new entity that we have introduced as a component of the universe, i.e., the spaceons; the two phases are indistinguishable. Hence, dark energy can be associated with spaceons, the energy of space. The amount of dark energy soon after the Big Bang was relatively small (Table 1) because most of the energy of space was converted to radiation, fundamental particles, dark matter and ordinary matter. Its density remains low as the universe expands.

The behavior of our model universe with spaceons is well suited to mathematical treatment using the Friedmann-Lemaitre-Robertson-Walker (FLRW) metric [11]. (Note: It is worthwhile to recall that in deriving his equations, Friedmann used an ideal gas as a model for his cosmic fluid.) The second Friedmann equation [10] [11] delineates the contributions of the various components of the universe (matter, radiation, and spaceons) to the accelerated expansion of the universe, i.e.,

$$
\mathrm{d}^{2} a / \mathrm{d} t^{2}=-(8 \pi G a / 3)\left\{(1 / 2) \rho_{m}+\rho_{r}-\Lambda / 8 \pi G\right\}
$$

We have set the curvature term, $k=0$, normally appearing in Equation (4), which corresponds to a Euclidean universe with a flat spacetime curvature. In the above equation, $a$ is the scale parameter, $G$ the gravitational constant; $\rho_{m}$ is the mass-energy density of matter, $\rho_{r}$ that of radiation, and $\Lambda$, the cosmological constant which represents the energy of the vacuum [10]. "Vacuum energy" is not a good term to use with our model, where the vacuum state is now the Void ("absolutely nothing"); it is preferable to use the term "energy of space". The cosmological constant will then be replaced by the energy density of space, $\rho_{s}$, i.e. 


$$
\mathrm{d}^{2} a / \mathrm{d} t^{2}=-(8 / 3) \pi G a / 2 \rho_{m}+\rho_{r}+\left(\rho_{s}+3 P_{s}\right)
$$

and $\rho_{s}$ is the energy density of space. It is related to the pressure $P_{s}$ via the equation of state $P_{s}=w_{s} \rho_{s}$ where $w_{s}$ is the equation of state parameter. A negative pressure would give rise to a positive $\rho_{s}$ that can cause an acceleration of the expansion.

For the purpose of fitting the observed data (Table 1), it is preferable to use another form of the Friedmnan equation. It is common to utilize one involving the Hubble constant, $H$, which is measured in experiments and use velocity, $\mathrm{d} a / \mathrm{d} t$, rather than acceleration [9] [10] [11]. Thus,

$$
a H^{2}=(\mathrm{d} a / \mathrm{d} t)^{2} a^{2}=\left(H_{0}\right)^{2}\left[\Omega_{m} a^{-3}+\Omega_{r} a^{-4}+\Omega_{s} a^{-3\left(1+w_{s}\right)}\right]
$$

$H=(\mathrm{d} a / \mathrm{d} t) \cdot a, H_{0}$ its value at the present time; $\varrho_{i}$ is the density parameter for component $i, \varrho_{c}$ is the critical energy density of the universe (the density of the universe at the present time) and $\Omega_{i}=\varrho_{i} / \varrho_{c}=f$, the fractional energy density.

A plot of fractional energy density as a function of of " $a$ " can be constructed to fit the measured composition of the universe at about the time of the Big Bang and at the present time (see Table 1). The plot in Figure 2 shows the evolution of the composition of the universe. The dominance of radiation, matter, and dark energy at different times are amply illustrated. As can be seen there is good fit of the data summarized in Table 1 at time $t=13.8$ billion years $(a=1)$ and at the time of the Big Bang, at about $t=380,000$ years $\left(a=5.25 \times 10^{-3}\right.$, redshift $z=$ 1089), with an equation of state parameter, $w_{s}=-0.7$. Dark Energy is very small in the early universe as most of the energy is used in the creation of radiation and fundamental particles. As the universe expands, however, the energy densities of radiation and matter continue to dilute and are eventually overcome by the

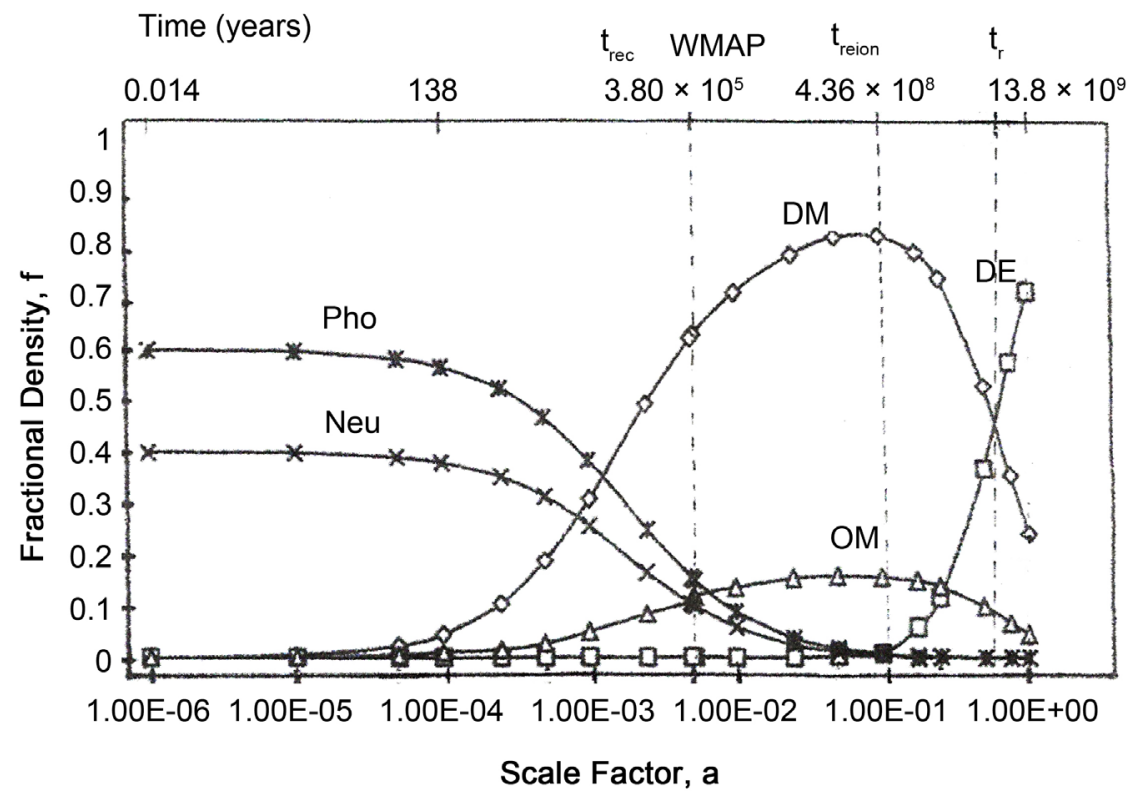

Figure 2. Fractional energy density $(f)$ vs scale factor $(a)$, fit with $w_{s}=-0.7$ DE-Dark energy; DM-Dark Matter; OM-Ordinary Matter; Pho-Photons; Neu-Neutrinos. 
energy density of Dark Energy. This occurs at around 7 billion years at which time the expansion of the universe starts to accelerate. We call this the transition time, $t_{r}$. This result has been established by Baryon Acoustic Oscillation measurements in the Baryon Oscillation Spectroscopic Survey (BOSS) project [13] [14] and supernova measurements [15]. Figure 3 lends support to these findings. One sees that the fractional energy density of Dark Energy (DE1) crosses that of total matter (TM1) at $t_{r}$ with $a=0.65$ ( $t=7.25 \times 10^{9}$ years). Siegel [16] has made a similar fit using a cosmological constant of -1 , but did not give a value for the transition time. Our calculation with a cosmological constant $(w=-1)$ yielded a time of $8.75 \times 10^{9}$ years $(a=0.74)$ for the dominance of dark energy (Figure 3), broken line, DE2, TM2). Result for the so-called Phantom Energy model [17] gives $w=-1.2$ for the transition time, $t_{r}$, and a later transition time of $9.2 \times 10^{9}$ years (DE3, TM3). The measurement of transition time, $t_{r}$, appears to be a good test for the suitability of models for Dark Energy.

The concept of a constant energy density for space leads to some difficulties. The cosmological constant is considered to be equal to the energy of the vacuum. Calculations give a 120 orders of magnitude for the calculated value of the density of dark energy; this is obviously wrong. Moreover, the constancy of dark energy in the course of cosmic evolution is thought to be unlikely [18]. It also poses difficulties in answering the question of where the energy comes from during the expansion of the universe. It is more reasonable to expect that the

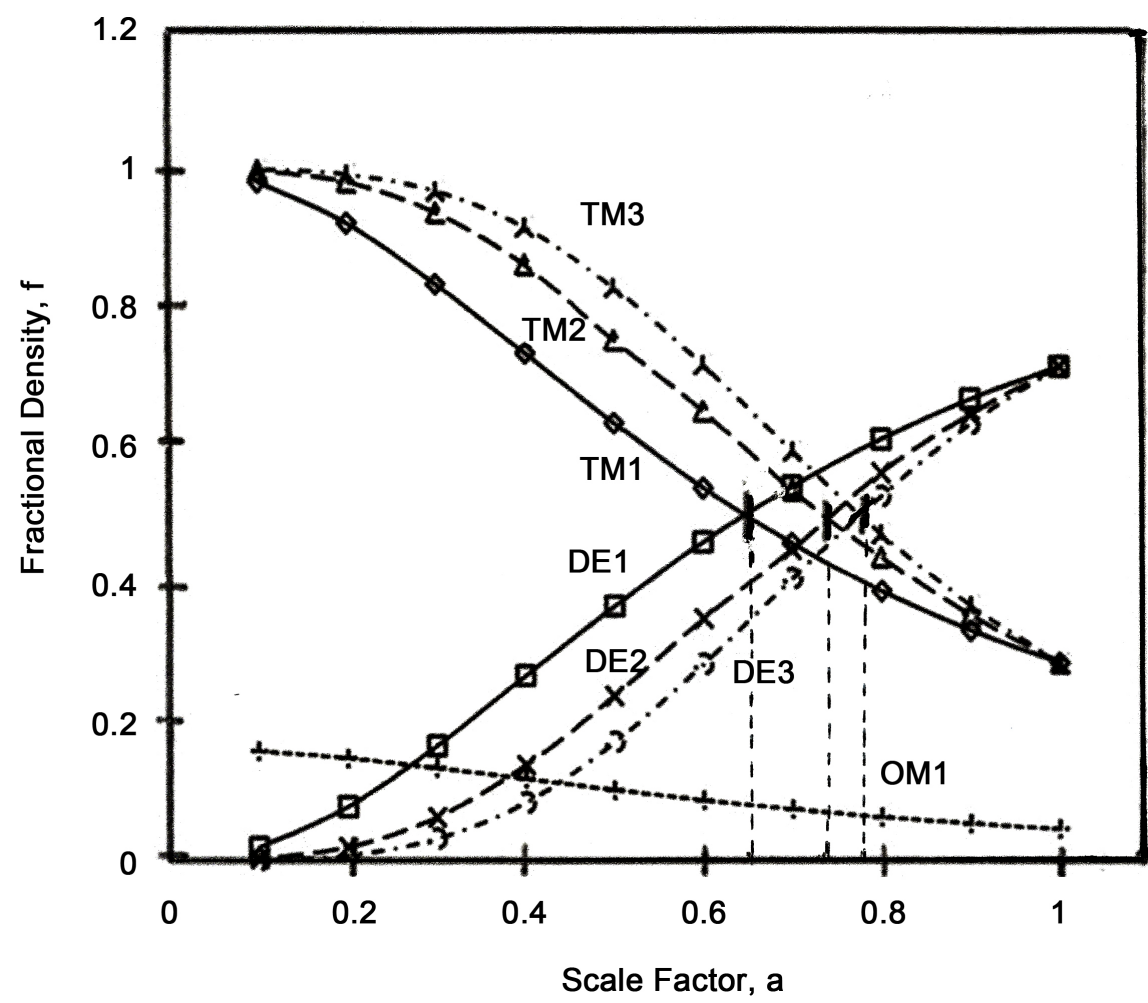

Figure 3. Fractional energy density (f) vs scale factor (a) DE1-Dark energy; DM1-Dark matter; OM1-Ordinary matter; TM1 - Total matter for $w_{s}=-0.7$; DE2, TM2 for fit with cosmological constant, $w=-1$; TM3, DE3 - for fit with phantom energy model, $w=-1.2$. 
energy density would dilute as the universe expands for all forms of energy. Quintessence [18], with spaceons as the cosmic fluid that is a component of the universe, appears to be the better explanation for dark energy.

\subsection{Thermodynamics of Expansion and Acceleration}

In our model, the universe started with a finite amount of energy. It was assumed to be an isolated system and expansion was adiabatic, with no energy transfer in and out of the universe. From thermodynamics,

$$
Q=\mathrm{d} E+P \mathrm{~d} V=0,
$$

where $Q$ is the energy flowing into or out of the system, $\mathrm{d} E$ the change in internal energy, $P$ the pressure, and $\mathrm{d} V$ the change in volume during the expansion. The work to create space, $P \mathrm{~d} V$, is done at the expense of the internal energy, i.e. $\mathrm{d} E=-P \mathrm{~d} V$ or

$$
\mathrm{d} E / \mathrm{d} V=-P,
$$

the slope of the $\mathrm{d} E / \mathrm{d} V$ plot is negative. One can also derive the relation,

$$
\begin{gathered}
H=\left(1 / 4 \pi r^{3}\right)(\mathrm{d} V / \mathrm{d} E)(\mathrm{d} E / \mathrm{d} t) \text { or } \\
\mathrm{d} E / \mathrm{d} t=4 \pi r^{3} H[\mathrm{~d} V / \mathrm{d} E]
\end{gathered}
$$

where, $r$, is the radius of the universe, and $H$ is the Hubble constant, proportional to expansion velocity. To a first approximation, a decrease in $H$ indicates a decrease in internal energy of the universe with time, $t$. BOSS studies of Busca et al. [ref [13], Figure 21], shows the expansion of the universe to be slowing down during the first 6 billion years after the Big Bang. A better plot is shown in Figure 4, from unpublished work by a Berkeley Group [14] who were collaborators in the Sloan Digital Sky Survey (SDSSIII) of the BOSS project. It showed that the expansion velocity reached a minimum around 7.5 billion years $\left(t_{r}\right)$. This is consistent with our model universe with the expansion being adiabatic initially, i.e. $\mathrm{d} E / \mathrm{d} t=$ negative. Turner and Riess [15] have also shown that the universe

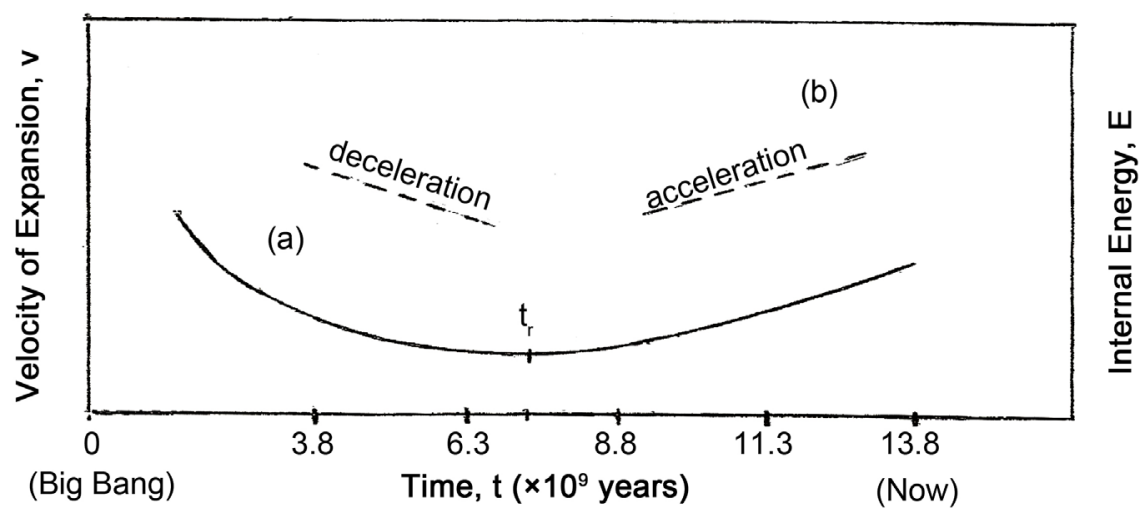

Figure 4. Rate of expansion of the universe as a function of time $(t)$ (Left Ordinate) tr-transition time from deceleration (a) to acceleration (b) (Right Ordinate) Schematic change in internal energy, $E$, with time; slope $=\mathrm{d} E / \mathrm{d} t$ ) (Figure is after Preuss, ref. [14] axes labels modified). 
changed from deceleration (during the matter dominated epoch) to acceleration at a redshift, $z=1$ or $t=7 \times 10^{9}$ years. The expansion velocity increased thereafter. The slope $\mathrm{d} E / \mathrm{d} V$ (and that of $\mathrm{d} E / \mathrm{d} t$ ) became positive at large $z$ (longer time, Figure 4(b)).

Thermodynamically,

$$
\mathrm{d} E / \mathrm{d} V=(\mathrm{d} Q / \mathrm{d} V)-P=+
$$

that is, $Q$ is no longer zero. The expansion has become non-adiabatic. This means an ingress of energy from outside the universe. Thermodynamics leaves the possibility that the accelerated expansion of the universe could occur with an "injection" or "leak" of energy from outside the universe, if it is open. However, there is no evidence at the present time that "something" exists outside the universe to support this. Thus, we take the universe to be closed and the expansion must remain adiabatic. Thermodynamics then demands that for the accelerated expansion to continue, the Pressure, $P$, in Equation (11) must be negative. It is interesting to note that this is just what is required by the Theory of Relativity and Friedmann's equation (Equation (4)). It is also the negative pressure associated with Dark Energy. This seems like a coincidence, but it is also required by the law of Conservation of Energy on which the Friedmann's equation is based [10]. Thus our model is totally consistent and most satisfying. It is important to note that the negative pressure cannot be taken as due to a repulsive form of gravity, as is often theorized.

We next try to answer the question of where the energy comes from to sustain the accelerated expansion by dark energy. The negative pressure means work is done on the system and energy is added to it. A likely source is the gravitational field through the action of gravity. The gravitational force is an attractive force. It exerts this force on all matter to clump together resulting in a decrease in gravitational potential energy, i.e., the negative potential energy becomes more negative. The gravitational energy lost in turn goes to increase the energy of space and the expansion to accelerate. A simple analogy is to imagine a rubber hose filled with running water. Squeezing the hose momentarily shrinks its diameter and causes water to squirt out. Figure 5(a) shows schematically the state of the universe during the period of deceleration $\left(5-7 \times 10^{9} \mathrm{yrs}\right)$ in the matter dominated epoch. Consider the cosmic fluid flowing through a tube (dash lines) within the universe. The Hubble flow (HF) is slowing down. The pressure of the universe is outward $\left(P_{s}=+\right)$ due to the inherent property of space (the spaceons) to expand into the Void; the slope $\mathrm{d} E / \mathrm{d} V$ is negative (Equation (7)). In Figure 5 (b), at the transition point, the attractive force of gravity starts to dominate with a pressure inward $\left(P_{g}=-\right) ; \mathrm{d} E / \mathrm{d} V$ becomes positive. In the process, the "cosmic fluid" contained in the tube is squeezed out much like that of the rubber hose. The fluid velocity or the Hubble flow increases, manifesting itself as an accelerated expansion of the universe.

During the expansion of the universe, following the Big Bang, matter (mostly atomic $\mathrm{H}$ gas) proceeded to consolidate and clump together to form stars, galaxies, 


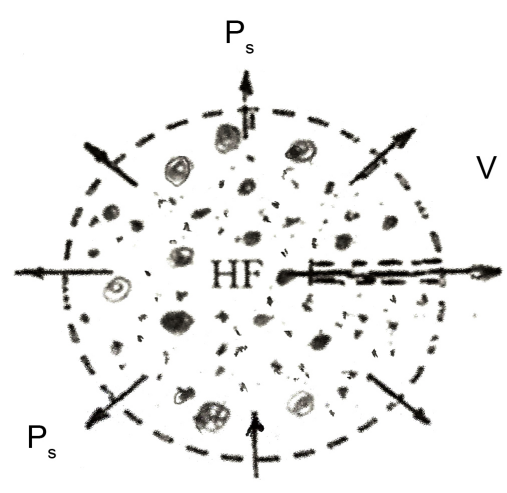

(a)

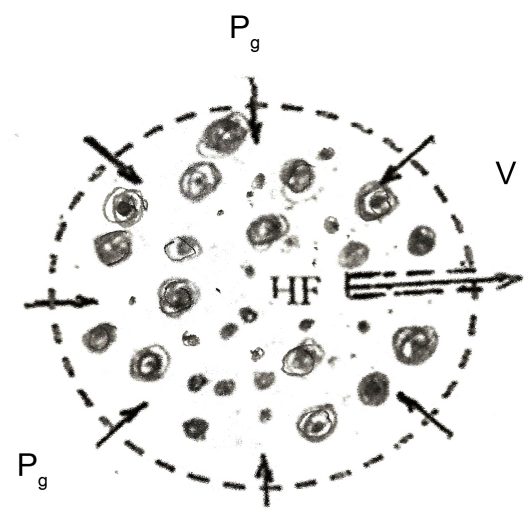

(b)

Figure 5. Schematic of the Universe during the period of deceleration (a) followed by acceleration (b); $P_{s}=+$, pressure due to space; $P_{g}=-$, pressure due to gravitational force; $\mathrm{HF}=$ Hubble flow; $\mathrm{V}=$ Void.

clusters, and superclusters. Through the action of gravity they eventually form black holes, neutron stars, and other massive compact objects. These grow by mergers and accretion of nearby materials (gas, dust, etc) which get compressed by gravity resulting in smaller volume of matter whose mass approaches infinite density, i.e., a Black Hole/neutron star. Chapline [19] [20] has questioned the reality of Black Holes and proposed an alternative theory where matter infalling into the event horizon, undergoes a phase transition into dark energy [20]. The theory resolves questions associated with black holes, one of which is the loss of information when matter is swallowed by the black hole singularity. It also explains other cosmic phenomena not explained by black holes. He suggested the name "Dark Energy Stars" for Black Holes. Likewise, Mazur and Mottola [21], as well as Barcelo et al. [22], have proposed similar theories and suggested the name Gravastars and Black Stars respectively. Rovelli et al. [23] [24], used Loop Quantum Gravity to show that a singularity can be prevented via a "big bounce" mechanism by which the gravitational pressure acting on matter is balanced by an opposite degeneracy-like pressure of the core. The latter eventually explodes, releasing energy and hence the information it contains; this solves the information loss paradox.

It is not really known what the real state of matter is in Planck stars; we theorize that further compression by gravity should ultimately transform "particulate" matter to quarks, gluons, and finally to spaceons (dark energy), per the theories of Chapline [19] [20] and Barcelo, et al. [22]. (This is consistent with our phase diagram in Figure 1, tracing the curve from low to high T). We will adopt a hybrid model incorporating features of the models of Chapline, Barcelo et al. and Rovelli. We suggest to use the more descriptive names "Quantum Stars", and "Space Well" for the singularity". Moreover, the singularity cannot be real but maybe just an artifact in the mathematics of General Relativity; such state cannot exist due to the fact that the volume of space must be finite and cannot be less 
than the Planck volume. (Our model indicates this as mentioned later in Section 5.) In any case, Black Holes or Quantum Stars will have an "equivalent massenergy" of $\mathrm{M}$. The gravitational potential energy of the universe becomes more negative due to much greater "masses" of the clumped massive compact objects compared to $\mathrm{H}$ atoms. One can calculate the total gravitational potential energy change between the present time $t_{p}$ and that at the time of transition, $t_{p}$, at the beginning of the accelerated expansion $\left(7.25 \times 10^{9}\right.$ years), for all pairs of compact and other objects ("masses") in the universe. The total energy at the present time, $t_{p}$, is

$$
U_{p}=-G\left[M_{i} \cdot M_{j} / R+m_{k} \cdot m_{l} / r+M_{i} \cdot m_{k} / s+\cdots\right],
$$

summed over pairs of all other masses, $m_{i, j, k, l, \ldots}$ (atoms, stars, etc) and Quantum Stars, other compact object, etc, $M_{i, j, k, l, \cdots}$ in the universe; $R, r, s, \cdots$ are their respective distances of pair separation. Similarly, we can calculate the total potential energy just before the transition time, $t_{r}$, to give $U_{r}$. The difference in potential energy, $\Delta U=U_{p}-U_{r}$, provides the energy that feed the further expansion of the universe. The calculation is unwieldy and is best done by simulation techniques.

Our proposed mechanism is supported by recent observations on the merger of 2 black holes or neutron stars. As the two massive bodies come together, their distance of separation decreases and in the process gravitational energy is released as gravitational waves [25]. The latter causes the perturbation of space that increases the energy of space and accelerates the expansion of the universe. This example explains the observed increase in internal energy vs time (slope, $\mathrm{d} E / \mathrm{d} t=+)$ as shown in Figure 4 .

\section{On the Nature of Dark Matter}

We now discuss the other major component of our invisible universe, dark matter [26]. It comprises $84 \%$ of the total mass of matter in the universe, or $24 \%$ of its total mass-energy. It remains mysterious. Among properties that have been deduced from observations are:

1) it neither emits or absorbs electromagnetic radiation, hence it is difficult to study.

2) it moves without friction.

3) it can only be detected through its gravitational effects on the motion of galaxies.

4) it is spread over large areas, like a cloud, and forms a "halo" around galaxies and clusters; its density decreasing as one moves away from the center [27]. The halo is consistent with a plasma which glows and is visible at large distances; ordinary matter is not luminous unless they generate their own light, like stars. It comes from the light that is unable to penetrate and escape out of the plasma cloud.

5) it is also found in filaments between galaxies and clusters [28]. It has been observed that ordinary matter traces the path of dark matter in these filaments; 
this has been attributed to a strong interaction between ordinary matter and dark matter [28].

We proceed with the premise that all components of the universe were formed during cosmic evolution and would have left their footprints in the sands of time, e.g., the $\mathrm{CMB}$ as a relic of the pre-recombination epoch. Table 1 and the phase diagram (Figure 1) show that the major phases in the formation of the universe are dark energy, baryonic or ordinary matter (gasses, solids), dark matter, and plasma. Any present-day component of the universe must have originated from one of these phases.

WMAP data (Table 1), show that dark matter constitutes the major component of the universe soon after the Big Bang. During this time, the universe consisted of a hot plasma of electrons, and ionized $\mathrm{H}$ and $\mathrm{He}$. It was opaque and cannot emit or absorb light. This state persisted until about 380,000 years after the Big Bang when recombination took place and the universe became transparent.

It can be seen from the plot in Figure 2 that during the period of recombination $\left(t_{\text {rec }}\right)$ until after reionization $\left(t_{\text {reion }}\right)$, the main constituent of the universe is Dark Matter. This corresponds to the period during which plasma existed (Table 2). From Figure 1, it can also be seen that the major phase present following nucleosynthesis is a plasma form of matter, i.e., $\mathrm{H}^{+}, \mathrm{He}^{+}$and free electrons. At this time the universe was opaque due to scattering of photons by free electrons and protons.

We thus see a strong correlation between Dark Matter and the plasma phase. The following properties of plasma [29] further support this contention:

1) Plasma, like dark matter, hangs around like a cosmic fog around galaxies and clusters, making it invisible and difficult to characterize.

2) That ordinary matter traces the path of dark matter is due to the fact that upon recombination of electrons and positive ions in the dark matter plasma, ordinary matter is formed; the plasma evaporates into $\mathrm{H}$ and $\mathrm{He}$ gasses. Thus ordinary matter follows the trail of dark matter ( 5 above). The web structure of the universe is due to clamping of ordinary matter, not dark matter.

3) Filamentation is a characteristic of plasmas; they move without friction, since the ions do not have attractive interaction and move collectively instead [30]. This lack of interaction also explains the "fluffininess" of dark matter halos [26] that hover around galaxies.

4) The dark matter plasma scatters elastically and hence do not clump or "stick together" thus remaining diffuse, fluffy, and "halo-like" for a long time. Hence, galaxies cannot form directly from dark matter.

It is therefore logical to make the conclusion that dark matter corresponds to the Plasma phase that existed during the photon epoch. We can then replace the Dark Matter in the phase diagram by Plasma. Plasma is the major form of matter in the universe [30], most of it is invisible and dispersed throughout the universe. This explains why "halos" are always found with dark matter along with 
ordinary matter at a ratio of about 5:1 from the Big Bang up to the present as found in Table 1 .

It is now generally accepted that Dark Matter resides in the Halos of galaxies and galaxy clusters. Halos are composed of gases, i.e., hydrogen and helium which are mostly ionized. This is consistent with the composition at the beginning of the universe. In addition to the WMAP composition measurements shown in Table 1, further evidences come from spectroscopic studies [31] [32] [33] [34] of hydrogen in the ancient universe and hydrogen/helium plasmas in the extreme ultraviolet and at radiofrequencies. They leave little doubt that Dark Matter is a plasma phase formed during the photon and reionization periods in the evolution of the universe. It is not surprising that Dark Matter plasma is itself a relic from near the $\mathrm{CMB}$ and during the reionization eras! Dark matter being a plasma form of matter seems closer to reality than exotic particles, like Weakly Interacting Massive Particles (WIMPs), Massive Astrophysical Compact Halo Objects (MACHOs), etc. [35]. Their existence has yet to be demonstrated despite innumerable experimental and theoretical work done to date.

Moreover, recent studies show that energy exchange between the dark matter plasma and the surrounding hydrogen provides a mechanism for interaction between the two states of matter [36]. The study of the enhanced recombination processes in plasmas [36] is yielding interesting results that support our theory of the origin of Dark Matter. They are worth pursuing along with spectroscopic studies of dark matter halos [31] [32] [33] [34].

\section{A Theory of Space and Space Quanta}

The nature of space, its quanta, and our Quantum Space model deserve further elaboration. The reality and nature of space and its quantization have not been discussed much in the scientific literature. It is treated like a canvas in which a portrait of the universe as a function of time, in effect, a film recording. We have a different concept. Space is all around us, it expands, it reacts to what it contains (matter, energy, radiation). It is a dynamical entity. It grows. It is part of our universe and plays a very important role in it. It obeys the Theory of General Relativity like an ordinary physical object, it exhibits length contraction. This is as it should be if it consists of energy quanta. As a participant in the evolution of the universe, we can follow and trace its progress and its ultimate fate.

As we noted earlier, Friedman's equations were derived using the model of an ideal gas as the cosmic fluid. Yet, the components of the universe were only radiation, matter, and something which he did not specify but we now refer to as "dark energy". We do not know what Friedmann had in mind when he created his "cosmic fluid", with a negative pressure which remains a mystery to us. Our model takes it as space, i.e., space quanta, which is something more physical.

Our Quantum Space model suggests and embodies some properties of space and space quanta:

1) Spaceons differ from electromagnetic radiation and do not carry a charge, 
hence they cannot be detected by spectroscopic techniques. Space also differs from gravity. Gravity is an attractive force between material objects. The space force is repulsive; it exerts a pressure opposite to that of gravity. It is inherent in space, is long range and weak, similar to gravity. In the language of Quantum Field Theory the space force is carried by spaceons which are bosons.

2) The space field is a scalar field. It is similar to the gravitational field. It differs from the vector field of electromagnetic waves. Recall that at the beginning of time, there were only energy and space. Therefore it seems logical to presume that the space field is the "mother" field, from which arose both force fields and matter fields [11] [12]. The space field has no charge. The electromagnetic field maybe just the space field with a charge. For that reason, it is formed early in the birth of the universe, along with spaceons. The notion that the Big Bang happened "everywhere with matter flying out everywhere" seems like an erroneus one; there is no evidence that matter existed at $10^{-12} \mathrm{sec}$ following the Big Bang. The fundamental particles (gluons and quarks) that made up matter were formed from the condensation of "hot" space quanta following the Quantum Space epoch. Thus an era of quantum space predates that referred to now as quantum gravity. Space, energy and time might be the most fundamental entities in nature; it is not matter. They are all convertible from one to the other. There cannot be anything without space; without space there is "nothing".

3) The spaceons propagate space and transport energy in the universe. From this energy emanate all radiation and matter. Space also acts as the container and reservoir of energy in the universe. Virtual particles can pop in and out of space. When particles and antimatter annihilate, they disappear and the energy goes back to space. The two are well established concepts in physics.

4) We might point out certain implications of our model and Equations (1)) and (2)).

At the beginning of time, the universe was point-size, $\mathrm{V}$ near 0 ; it carried almost infinite amount of energy, its temperature also almost infinitely high. In the "end", $\mathrm{V}$ will approach infinity as space continues to expand; its total energy will approach 0 , its temperature nearing absolute $0 \mathrm{~K}$. Since the universe will never reach these "endpoints", the universe cannot have arisen from a "singularity" as people claim to be our beginning. Likewise, the universe cannot completely end in "oblivion" or "absolute nothingness". It could be that our universe is cyclic and infinite; there may have been Big Bangs before ours.

5) Finally, we may comment on the geometry of the universe as this topic is also quite controversial. It is widely accepted that the universe is "flat" and probably open, so that the law of conservation of energy is not obeyed [37]. The latter is a principle that forms a pillar of our scientific foundation; it would be extremely difficult to tear down. Th universe is also likely to be infinite in extent. The flatness has been challenged by Di Valentino, Silk and others who proposed a closed, spherical universe [38]. The idea goes against conventional thinking, but our model supports that view. 


\section{Conclusion}

A physico-chemical approach, using a Quantum Space model and thermodynamics, appears useful in understanding the expansion and composition of our dark universe. Dark energy is the energy of space and the cosmic fluid that is the component of the universe responsible for its expansion. It maybe thought of as a scalar space field, dubbed as Quintessence. Dark matter, on the other hand, is a plasma form of matter, similar to the state of the universe at the photon epoch, before recombination and during reionization. Thus it is a relic of nearly the same period as the CMB. Dark energy and dark matter are neither "dark" nor "mysterious", they are just invisible; one is transparent, while the other is opaque. Further work is necessary to better understand the nature and properties of the quantum space field and spaceons. Spectroscopic studies, along with those of astrophysical plasmas, would be a good way to further understand the nature of dark matter. The Theory of Inflation appears unnecessary to produce a homogeneous and isotropic universe; the continuity of space assures these. Finally, thermodynamics indicates that the acceleration in expansion of our universe is not due to a repulsive form of gravity. It requires the universe to be closed and the expansion adiabatic with a negative pressure as is necessary for energy conservation, consistent with the Theory of General Relativity and Friedmann's equation. We provide a mechanism to explain the acceleration in Hubble Flow as due to the decrease in gravitational potential energy of the universe resulting from the clumping and consolidation of matter; this feeds back to the energy of space and accelerates the expansion. Our Quantum Space model describes well the behavior of our universe and cosmic evolution. It sheds light on the "mysteries" of our dark universe, i.e., the nature of dark matter and dark energy; it makes a hypothesis on its origin and predictions on its origin and future fate. It needs further quantification, perhaps by using methods of Quantum Field Theory; and it may be useful in unifying quantum behavior and gravity in a Theory of Quantum Gravity.

\section{Conflicts of Interest}

The author declares no conflicts of interest regarding the publication of this paper.

\section{References}

[1] Futch, M. (2008) Leibniz's Metaphysics of Time and Space. Springer, Berlin. https://doi.org/10.1007/978-1-4020-8237-5

[2] Jaffe, R.L. (2005) Physical Review D, 72, Article ID: 021301. https://doi.org/10.1103/PhysRevD.72.021301

[3] Atkins, P. and De Paula, J. (2014) Physical Chemistry. W.H. Freeman, New York.

[4] Silk, J. (1989) The Big Bang. W.H. Freeman, New York.

[5] Weinberg, S. (1977) The First Three Minutes. Basic Books, New York.

[6] Delsemme, A. (1998) Our Cosmic Origins. Cambridge University Press, Cambridge. 
https://doi.org/10.1017/CBO9780511549199

[7] Bennett, C.L., Larson, L., Weiland, J.L., Jarosk, N., et al. (2013) The Astrophysical Journal Supplement, 208, 20. https://doi.org/10.1088/0067-0049/208/2/20

[8] Kirkwood, J.G. and Oppenheim, I. (1961) Chemical Thermodynamics. McGraw-Hill, New York.

[9] Frieman, J.A., Turner, M.S. and Huterer, D. (2008) Annual Review of Astronomy and Astrophysics, 46, 385-432.

https://doi.org/10.1146/annurev.astro.46.060407.145243

[10] Stenger, V.J. (2006) The Comprehensible Cosmos. Prometheus Books, New York.

[11] Weinberg, S. (2014) Cosmology. Oxford University Press, Oxford.

[12] Zee, A. (2010) Quantum Field Theory in a Nutshell. 2nd Edition, Princeton University Press, Princeton.

[13] Busca, N.G., Delubac, T., Rich, J., et al. (2013) Astronomy and Astrophysics, 552, A96.

[14] Preuss, P. (2012) BOSS Quasars Unveil a New Era in the Expansion History of the Universe. https://newscenter.lbl.gov/2012/11/12/boss-quasars-early-universe

[15] Turner, M. and Riess, A. (2002) Astrophysical Journal, 569, 18-22. https://doi.org/10.1086/338580

[16] Siegel, E. (2016) Personal Communication.

[17] Caldwell, R.R. (2002) Physics Letters B, 545, 23-29. https://doi.org/10.1016/S0370-2693(02)02589-3

[18] Zlatev, I., Wang, L. and Steinhardt, P. (1999) Physical Review Letters, 82, 896-899. https://doi.org/10.1103/PhysRevLett.82.896

[19] Chapline, G. (2005) Dark Energy Stars. Proceedings of the Texas Symposium on Relativistic Astrophysics, Stanford, December 2004, 101.

[20] Chapline, G., Hohlfeld, E., Laughlin, R.B. and Santiago, D.I. (2003) International Journal of Modern Physics A, 18, 3587-3590. https://doi.org/10.1142/S0217751X03016380

[21] Mazur, P.O. and Mottola, E. (2004) Proceedings of the National Academy of Sciences, 101, 9545-9550. https://doi.org/10.1073/pnas.0402717101

[22] Barceló, C., Liberati, S., Sonego, S. and Visser, M. (2008) Physical Review D, 77, Article ID: 044032. https://doi.org/10.1103/PhysRevD.77.044032

[23] Barrau, A. and Rovelli, C. (2014) Physics Letters B, 739, 405-409. https://doi.org/10.1016/j.physletb.2014.11.020

[24] Haggard, H. and Rovelli, C. (2015) Physical Review D, 92, Article ID: 104020. https://doi.org/10.1103/PhysRevD.92.104020

[25] Abbott, B.P., et al. (2016) Physical Review Letters, 116, Article ID: 06110.

[26] Trimble, V. (1987) Existence and Nature of Dark Matter in the Universe. Annual Review of Astronomy and Astrophysics Vol. 45, Annual Reviews, Inc., Palo Alto, 425-472. https://doi.org/10.1146/annurev.aa.25.090187.002233

[27] De Rujula, A., Jetzer, P. and Masso, E. (1992) Astronomy and Astrophysics, 254, 99-104.

[28] Dietrich, J., et al. (2012) Nature, 487, 202-204. https://doi.org/10.1038/nature11224

[29] Adachi, I., et al. (2020) Physical Review Letters, 121, Article ID: 141801.

[30] Gurnett, D.A. and Bhattacharjee, A. (2005) Introduction to Plasma Physics: With Space and Laboratory Applications. Cambridge University Press, Cambridge. 
https://doi.org/10.1017/CBO9780511809125

[31] Labov, S.E. and Bowyer, S. (1991) Astrophysical Journal, 371, 810-819. https://doi.org/10.1086/169946

[32] Mills, R.L. and Ray, P. (2002) International Journal of Hydrogen Energy, 27, 301-322. https://doi.org/10.1016/S0360-3199(01)00116-1

[33] Bowman, D., Rogers, A., et al. (2018) Nature, 855, 67-70. https://doi.org/10.1038/nature25792

[34] Barkana, R. (2018) Nature, 555, 71-74. https://doi.org/10.1038/nature25791

[35] Griest, K. (1997) Annals of the New York Academy of Sciences, 688, 390-407. https://doi.org/10.1111/j.1749-6632.1993.tb43912.x

[36] Nemer, A., Sterling, N.C., Raymond, J., et al. (2019) The Astrophysical Journal Letters, 887, L9. https://doi.org/10.3847/2041-8213/ab5954

[37] Josset, T., Perez, A. and Sudarsky, D. (2017) Physical Review Letters, 118, Article ID: 021102. https://doi.org/10.1103/PhysRevLett.118.021102

[38] Di Valentino, E., Melchiorri, E. and Silk, J. (2020) Nature Astronomy, 4, 196-203. https://doi.org/10.1038/s41550-019-0906-9 